\title{
A DISCRETE NON-LOCAL (DNL) OUTGOING BOUNDARY CONDITION FOR DIFFRACTION OF SURFACE WAVES
}

\author{
R. P. BONET,* N. NIGRO, M. A. STORTI AND S. R. IDELSOHN \\ Grupo de Tecnología Mecánica del INTEC, Güemes 3450, 3000 - Santa Fe, Argentina
}

\begin{abstract}
SUMMARY
A discrete non-local (DNL) boundary condition is used to solve the water waves propagation problem over variable depth. This condition is obtained by means of full solution of the discrete Helmholtz operator in a structured network. We consider a simulation of wave propagation around a circular island located on either a paraboloidal shoal or constant depth bathymetry. Such examples confirm the important improvement in accuracy for the DNL method over standard conditions in the near field. C) 1998 John Wiley \& Sons, Ltd.
\end{abstract}

KEY WORDS outgoing boundary condition; Berkhoff; discrete; non-local; surface waves; scattering

\section{INTRODUCTION}

When a long wave (for example, a tsunami) is propagating over waters of variable depth, it may be greatly amplified due to the variation of sea bed topography and/or at the coast; this is a highly non-linear phenomenon. The scattering of waves by a circular island located on a paraboloidal shoal is a well-known problem of long wave propagation; ${ }^{1-11}$ (see Figure 1).

The calculation of diffraction of water waves over a varying sea bed, based on finite element methods, was done by Berkhoff ${ }^{12}$ and Chen and Mei ${ }^{13-15}$ initially. Chen and Mei used a FourierBessel expansion as an exterior solution in a wave diffraction problem and a specially devised variational statement to link the exterior solution with finite element solutions in the interior domain.

Zienkiewicz et al..$^{6-9}$ made an important contribution in this way, when they proposed a general methodology for the solution about this problem. There were several strategies which allowed one to link finite element solutions to any kind of Helmholtz equation exterior solution (analytical, series or boundary integral).

This problem has also been solved by Tsay and Liu, ${ }^{16}$ Houston, ${ }^{17} \mathrm{Xu}$ et al. ${ }^{18}$ and Bonet, ${ }^{19}$ who incorporated the exact radiation condition at infinity in the numerical scheme by means of a 'sponge-filters' method.

Different procedures based on 'infinite elements' to solve the exterior problem governed by the Helmholtz equation on an unbounded domain are given by Zienkiewicz and Bettess ${ }^{10,11}$ and H. S. Chen. ${ }^{20}$ They used of 3- and 2-node shape functions, respectively, to approximate the

* Correspondence to: R. P. Bonet, Grupo de Tecnologia Mecania, INTEC, Guemes 3450, 3000 Santa Fe, Argentina. E-mail: rbonet@venus.unl.edu.ar

Contract grant sponsor: CONICET, Argentina; BID 802/OC-AR PID 26.

Contract grant sponsor: Universidad Nacional del Litoral, Argentina.

CCC 1069-8299/98/090849-13\$17.50

Received 16 September 1997

(C) 1998 John Wiley \& Sons, Ltd.

Accepted 13 February 1998 


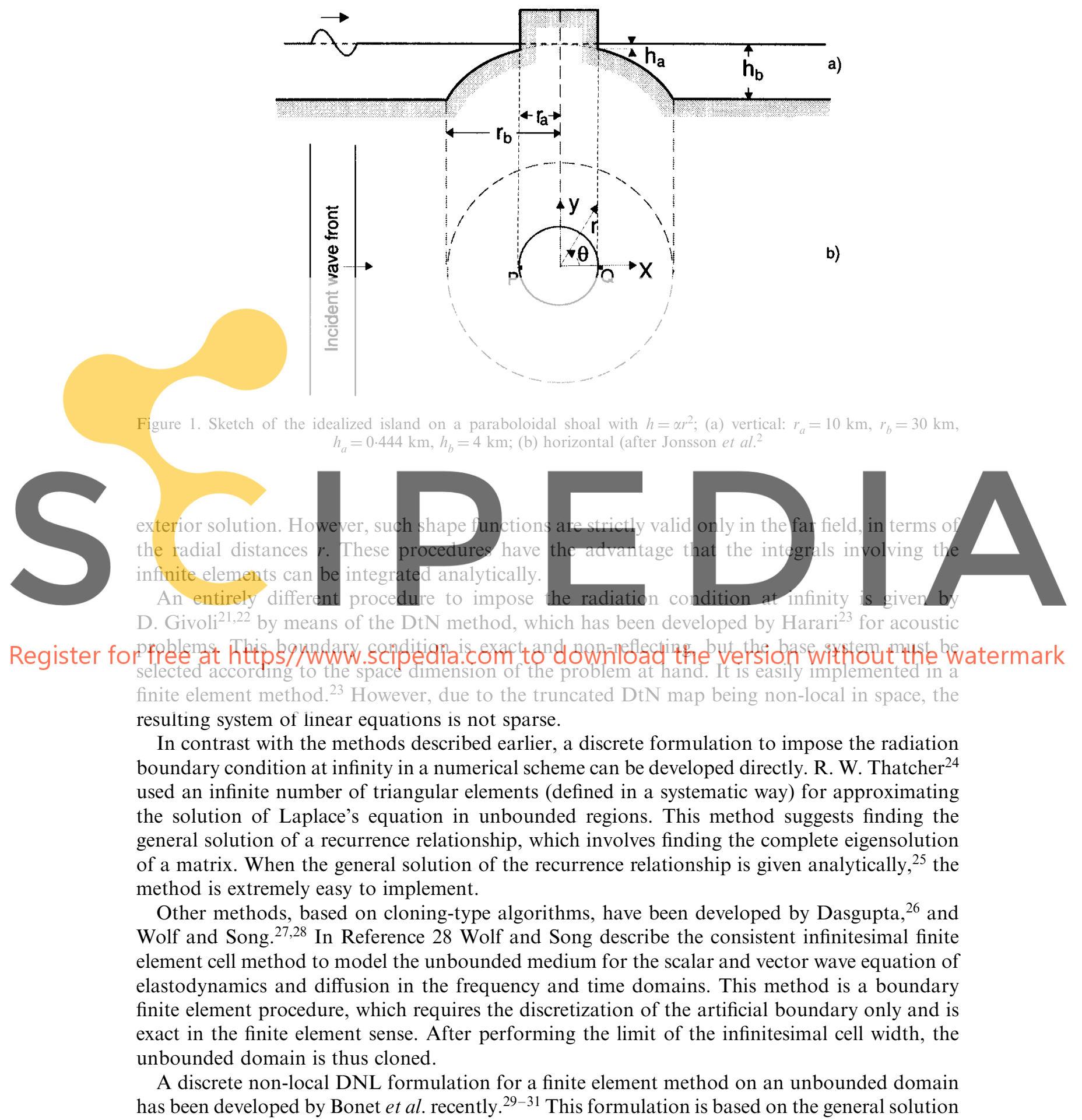


of a recurrence relationship which involves the complete eigendecomposition of the discretized operator (as well as Thatcher's method ${ }^{24}$ ) over a structured mesh with quadrangular linear elements. This formulation, like the consistent infinitesimal finite element cell method, ${ }^{28}$ requires discretization on the artificial boundary only. The planar DNL boundary condition is non-local in space (as well as the truncated DtN map) and non-reflective.

In this paper we employ a formulation for a bounded computational domain that are derived by the DNL method for the circumferential case. The DNL procedure represents a general methodology. It has been applied to solve exterior problems associated with the Helmholtz operator, ${ }^{29,31}$ employing the finite difference (FDM) or finite element (FEM) method, as well, in the solution of the ship wave resistance problem. ${ }^{32}$ By means of this procedure the exterior problem associated with the Berkhoff equation ${ }^{12}$ is solved numerically, and a DNL boundary condition over the artificial boundary is obtained. This condition has the ability to describe scattered waves in all directions adequately, yet in the near field.

We present the shallow water diffraction solution for the scattering of water waves by a circular island and we compare the cases when the island is and is not located on a paraboloidal shoal. These numerical tests validate the numerical method employed.

\section{FORMULATION}
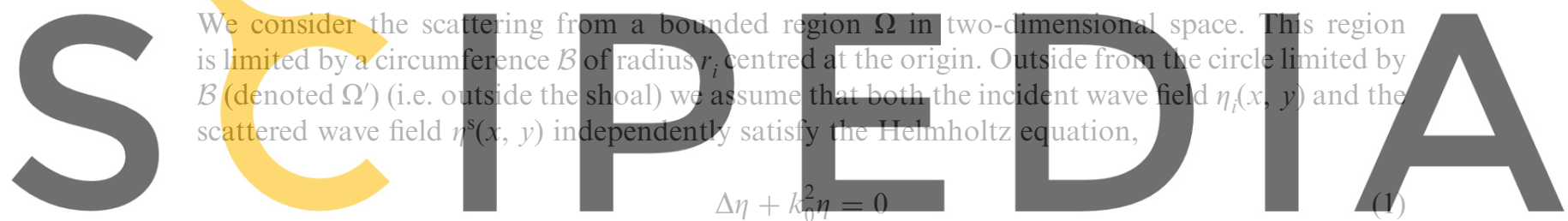

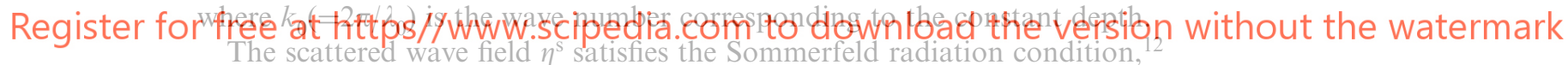

$$
\lim _{r \rightarrow \infty} \sqrt{\left(k_{0} r\right)}\left(\frac{\partial}{\partial r}-i k_{0}\right) \eta=0
$$

We suppose that the domain $\Omega$ within $\mathcal{B}$ is bounded internally by the smooth surface $\Gamma$ (see Figure 2). In the region with variable water depth (i.e. in the region over the shoal) the timeindependent part of the water elevation $\eta(x, y)$ satisfies the long-wave equation ${ }^{2}$

$$
\nabla(h \nabla \eta)+k^{2} h \eta=0 \text { in } \Omega
$$

where $\nabla$ is the horizontal gradient operator, $\omega$ is the angular frequency, $g$ is the gravitational acceleration, and $h=h(x, y)$ is the water depth. $\left.C=C_{g}=\sqrt{(} g h\right)$ are the phase and group velocity, respectively; $k=\omega / C \geqslant 0$ is the wave number, such that $k(x, y)$ changes continuously until to the boundary $\mathcal{B}$, where it takes the value $k_{0}$. (This theoretical formulation is classical and you can find it expressed in other papers, of different forms, for example in References 2, 9 and 26.)

In the external region to $\Omega$ the numerical solution is derived by means of a partial discretization process. Details are included in the following Sections. 

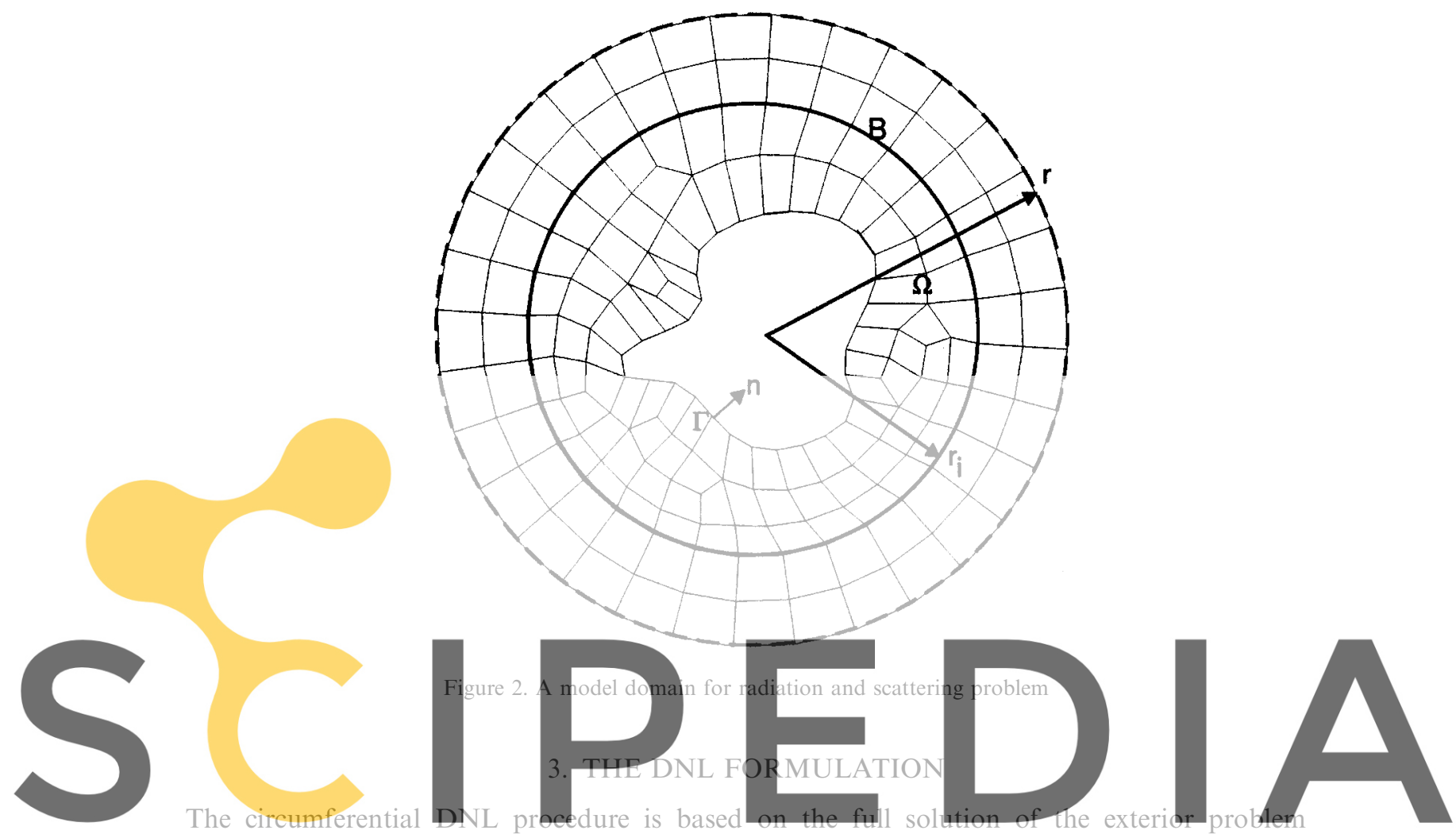

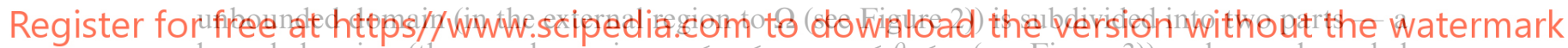
bounded region (the annular region $r_{\mathrm{i}} \leqslant r \leqslant r_{\mathrm{e}},-\pi \leqslant \theta \leqslant \pi$ (see Figure 3)) and an unbounded semi-infinite region (the region $r \geqslant r_{\mathrm{e}}$ ). Figure 3 (left) shows the scatterers surrounded by a circle
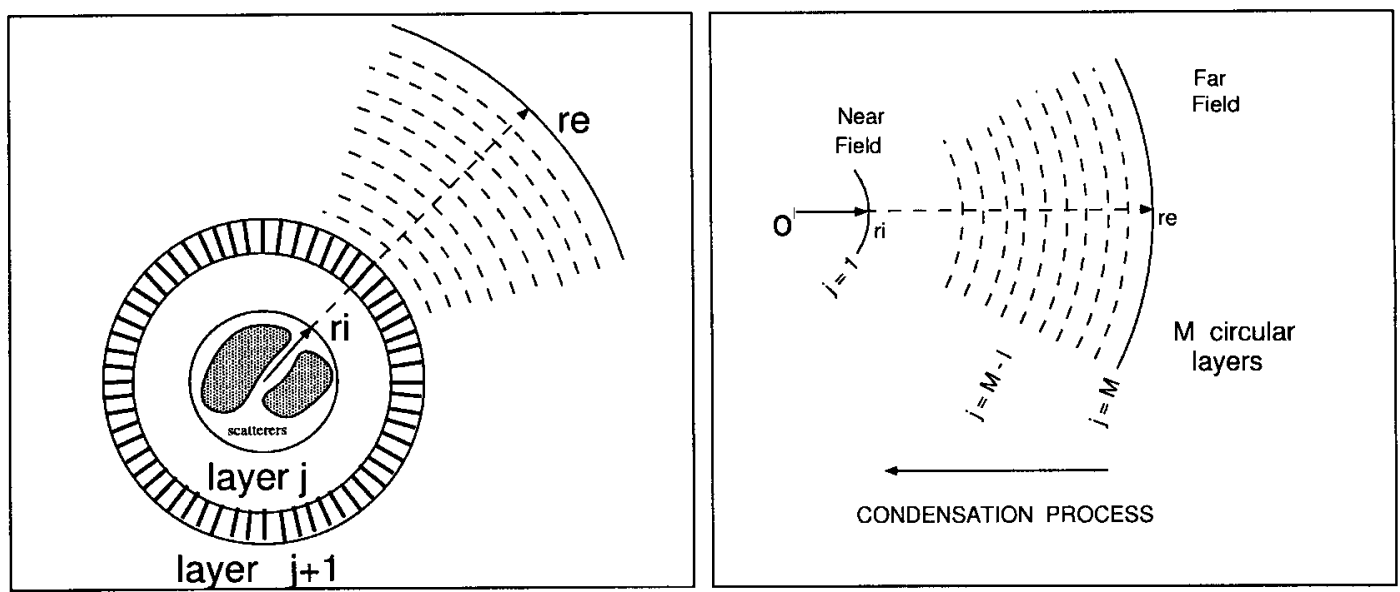

Figure 3. Circular DNL method: (left) sketch on the scattering process by DNL method; (right) condensation process 
of radius $r_{\mathrm{i}}$, located at the artificial boundary (in the near field) and the successive circles (0 layers) until the circle $r=r_{\mathrm{e}}$ (in the far field), which is taken sufficiently large such that the influence of curvature can be neglected. In contrast with Thatcher's method, ${ }^{24}$ the DNL method uses a discretization with quadrangular linear elements (see layer $j$ in Figure 3), resulting in extremely easy implementation. The crucial issue is, first, the adequate representation of the scattered wave field in the far field $\left(r \geqslant r_{\mathrm{e}}\right.$ ), and second, in the near field (where is located the artificial boundary of the computational domain $\left(r=r_{\mathrm{i}}\right)$ ).

We describe here briefly such a procedure performing the partial discretization of the Helmholtz equation (1) in polar co-ordinates, with quadrangular linear elements. Integrating in the transversal direction $\theta$, we obtain a second order differential equation system in $r$ of the form

$$
\mathbb{I} \ddot{\eta}+\frac{1}{r} \mathbb{I} \dot{\eta}-\frac{1}{r^{2}} \mathbf{M}^{-1} \mathbb{K} \eta+k_{0}^{2} \mathbb{I} \eta=\overline{0}
$$

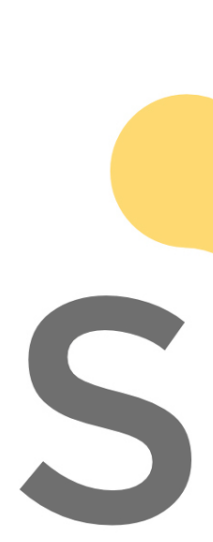

where $\eta=\eta^{\mathrm{s}}, \dot{\eta}=\mathrm{d} \eta^{\mathrm{s}} / \mathrm{d} r, \mathbf{M}$ and $\mathbf{K}$ are the mass and stiffness assembled matrices, respectively, and $\boldsymbol{I}$ is the identity matrix. Discretization of equation (4) in $r$ can be done by the finite difference method or the finite element method. Independently of the partial discretization procedure employed, the corresponding discrete equation for layer $j$ has the form

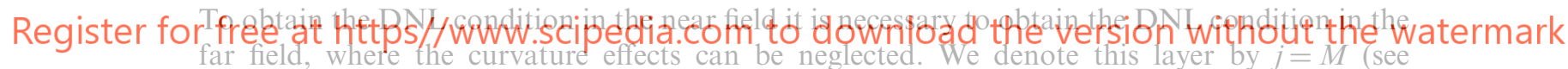

Figure 3). For layer $j=M(5)$ reduces to

$$
\mathbf{A}^{M} \eta^{M-1}+\mathbf{B}^{M} \eta^{M}+\mathbf{A}^{M} \eta^{M+1}=0
$$

The $\mathbf{A}^{M}$ and $\mathbf{B}^{M}$ matrices are real, and cyclic in virtue of their periodicity. Their dimension is $N_{\text {lay }} \times N_{\text {lay }}$. We consider that the $\mathbf{A}^{M}$ and $\mathbf{B}^{M}$ matrices remain almost constant for the layer $j \geqslant M$ (in the semi-infinite region $r \geqslant r_{\mathrm{e}}$ ), and it allows the application of the planar DNL method. ${ }^{30}$ Then, we denote $\mathbf{A}^{M}=A$ and $\mathbf{B}^{M}=B$ for the layer $j \geqslant M$.

For the real matrix $A^{-1} B$, there is an orthogonal transformation $V$, such that

$$
A^{-1} B=V \Lambda V^{-1}
$$

where $\Lambda=\operatorname{diag}\left(\lambda_{1}, \lambda_{2}, \ldots, \lambda_{N_{\text {lay }}}\right)$ is a diagonal matrix formed by the eigenvalues of the $A^{-1} B$ matrix and $V$ is the eigenvector system of $A^{-1} B$.

By means of the non-singular transformation

$$
\eta_{l}^{j}=\sum_{i=1}^{N_{\text {lay }}} V_{l, i}\left(c_{i}^{+} \mu_{i}^{+j}+c_{i}^{-} \mu_{i}^{-j}\right), \quad l=1,2, \ldots, N_{\text {lay }}
$$


the scattered wave field is split into 'forward' and 'backward' propagation modes, where $\mu_{i}^{ \pm}$is the characteristic equation solution:

$$
\left(\mu_{i}^{2}+\lambda_{i} \mu_{i}+1\right)=0
$$

In previous work the propagation modes were characterized, as for each $\lambda_{i}$ the roots $\mu_{1}, \mu_{2}$ of (9) satisfy $\left|\mu_{1}\right|\left|\mu_{2}\right|=1$; then two possibilities arise. If $\left|\mu_{1}\right|<1<\left|\mu_{2}\right|$, then we define $\mu_{i}^{+}=\mu_{1}$, $\mu_{i}^{-}=\mu_{2}$, whereas if $\left|\mu_{1}\right|=\left|\mu_{2}\right|=1$, the selection is done on the basis of the group velocity, which results in $\operatorname{Im}\left(\mu_{i}^{+}\right)>0 .{ }^{30}$

We denote by $G$ the matrix $G=\operatorname{diag}\left(\mu_{1}(\lambda), \ldots, \mu_{N_{\text {lay }}}(\lambda)\right)$ and based on equation (8), it is easy to prove that equation (6) is satisfied exactly for 'forward' propagation modes by means of the relation

$$
\left(\eta^{+}\right)^{j+1}=\mathbf{F}\left(\eta^{+}\right)^{j}
$$

such that the $F$ matrix named the planar DNL matrix can be given by $\mathbf{F}=V G V^{-1}$

Then, by means of this procedure developed for the rectangular Cartesian co-ordinates, ${ }^{29,30}$ we obtain the corresponding DNL matrix $\mathbf{F}^{M}=\mathbf{F}$ for the layer $j=M$ :
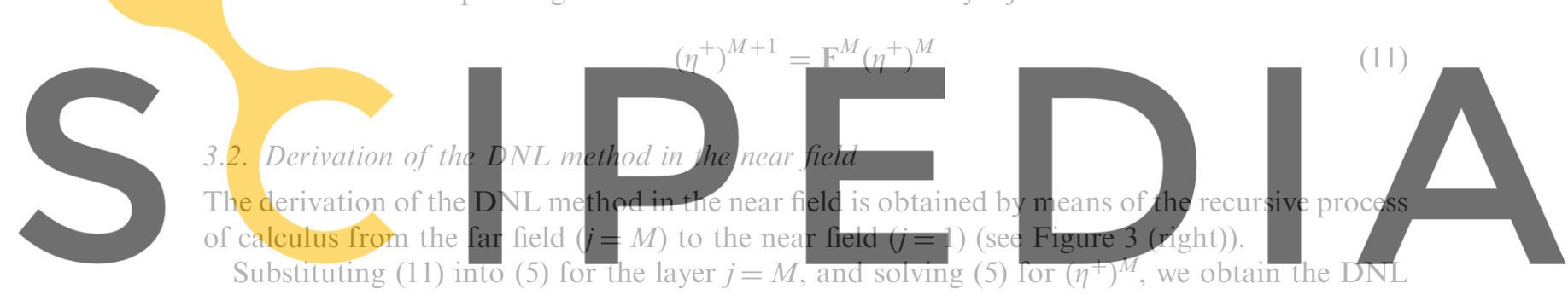
matrix for the layer $j=M-1$

Register for free at https//www.scipedia.com to download the version without the watermark

$$
\mathbb{F}^{M-1}=-\left(\mathbf{A}^{M} \mathbb{F}^{M}+\mathbb{B}^{M}\right)^{-1} \mathbf{C}^{M}
$$

Repeating recursively this calculation from layer $j=M-1$ to $j=1$ (the layer corresponding to $r=r_{\mathrm{i}}$ ) we obtain the DNL matrix for layer $r=r_{\mathrm{i}}$ and the relation

$$
\left(\eta^{+}\right)^{2}=\mathbf{F}^{1}\left(\eta^{+}\right)^{1}
$$

on the artificial boundary $r=r_{\mathrm{i}}$. This relation in the near field represents a discrete non-local solution of the exterior problem governed by the Helmholtz equation. We note that the $F^{1}$ matrix contains all the information on the behaviour of the scattered wave field since the far field.

This process is numerically stable and is named 'condensation from the plane DNL matrix to the circular DNL matrix'.

We note that the solution of the exterior problem (1)-(2) is influenced by the location of the $r=r_{\mathrm{e}}$ external boundary, where is calculated the rectangular DNL condition. In previous work Bonet et $a .^{31}$ verified that the scattering errors along the $r=r_{\mathrm{i}}$ boundary will diminish as the circle $r=r_{\mathrm{e}}$ is placed far away from the computational domain. In this process, the CPU time increases with the exterior radius $r_{\mathrm{e}}$, without increasing the RAM memory. We recall that, in general, these kinds of applications are memory bounded. Furthermore, the number of operations can be drastically reduced by means of an eigenvalue decomposition of the matrix $\mathbf{M}^{-1} \mathbf{K}$. 
Such a procedure has been developed for the study of radiation and scattering water waves around a circular island. ${ }^{29,31}$

\section{NUMERICAL RESULTS}

\subsection{Scattering of a plane wave from a circular island}

We have computed the scattering of an incident plane wave travelling along the positive $x$-axis $(\theta=0)$ (by a circular island of radius $r_{a}$ and depth $\left.=4 \cdot 0 r_{a}\right)$, in a normal direction to the cylinder's axis. We consider a hard boundary along the coast and the DNL boundary condition at the far boundary. With a condensation width of 8 wavelengths the numerical results are presented.

Figure 4 shows the absolute values of the analytical/numerical solution, for different values of radius $R=r_{a}, 2 r_{a}$ and $3 r_{a}$. In this Figure, the numerical solutions represent the expected physical behaviour of the solution. Numerical solutions about this problem have been reported by Zienkiewicz et $a l .{ }^{9,10}$ for the relation depth $=5 \cdot 0 r_{a}$, and by $\mathrm{Chen}^{20}$ and Berkhoff ${ }^{12}$ for the relation $k r_{q}=2 \cdot 0$.

\subsection{Diffraction calculations for waves incident on an island located on a paraboloidal shoal}

An island of circular cylindrical shape, located on a paraboloidal shoal in an infinite ocean of constant depth is attacked by small amplitude regular waves of long period and of plane

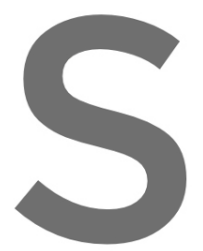
incidence (see Figure 1) theory. The bathymet accepted as being rep
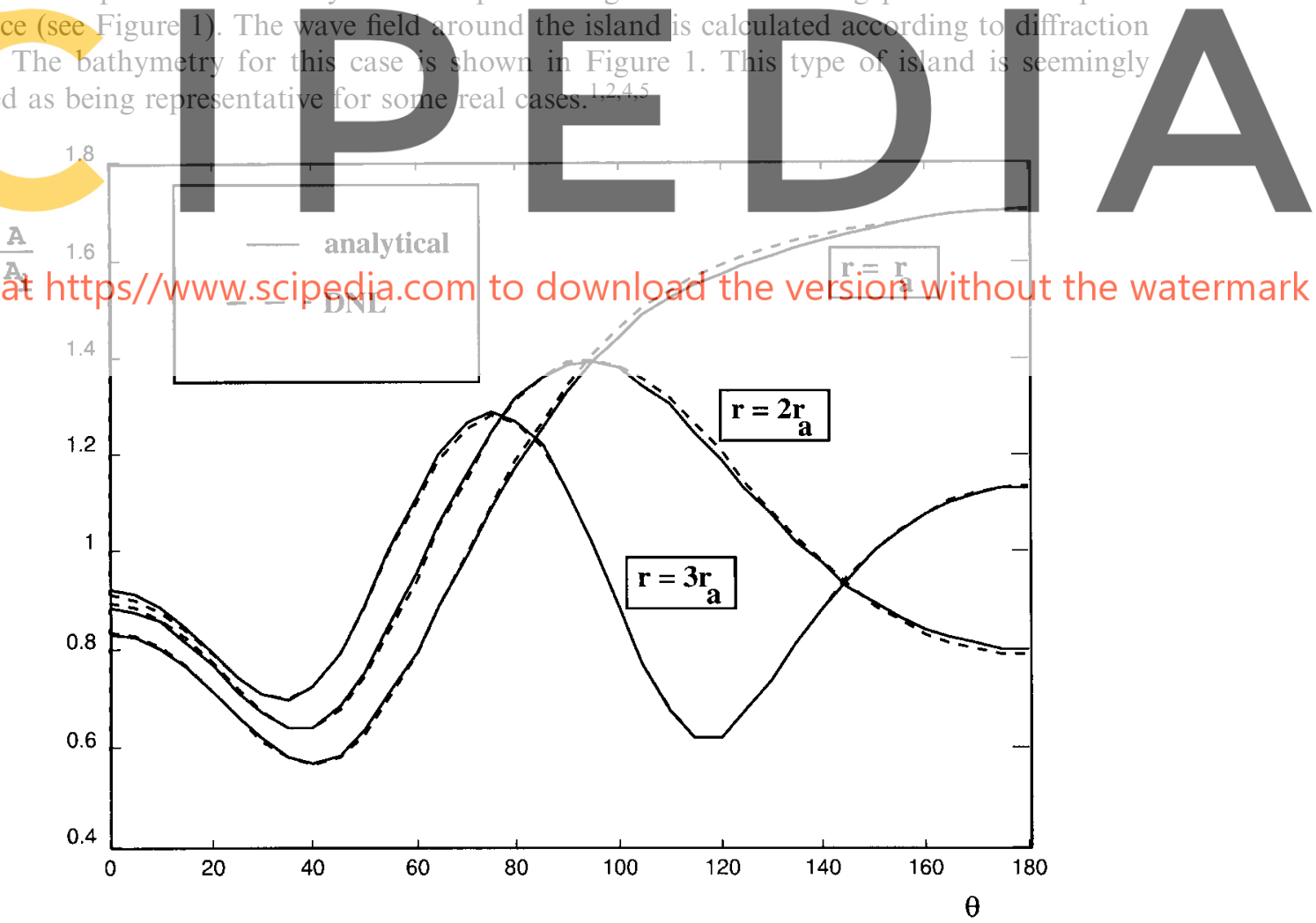

Figure 4. Scattering of a plane wave (at $\theta=0$ ) from a circular island of radius $r_{a}, k r_{a} \cong 1 \cdot 23$. Absolute values of the nodal interpolation of the series solution and the Galerkin solution 

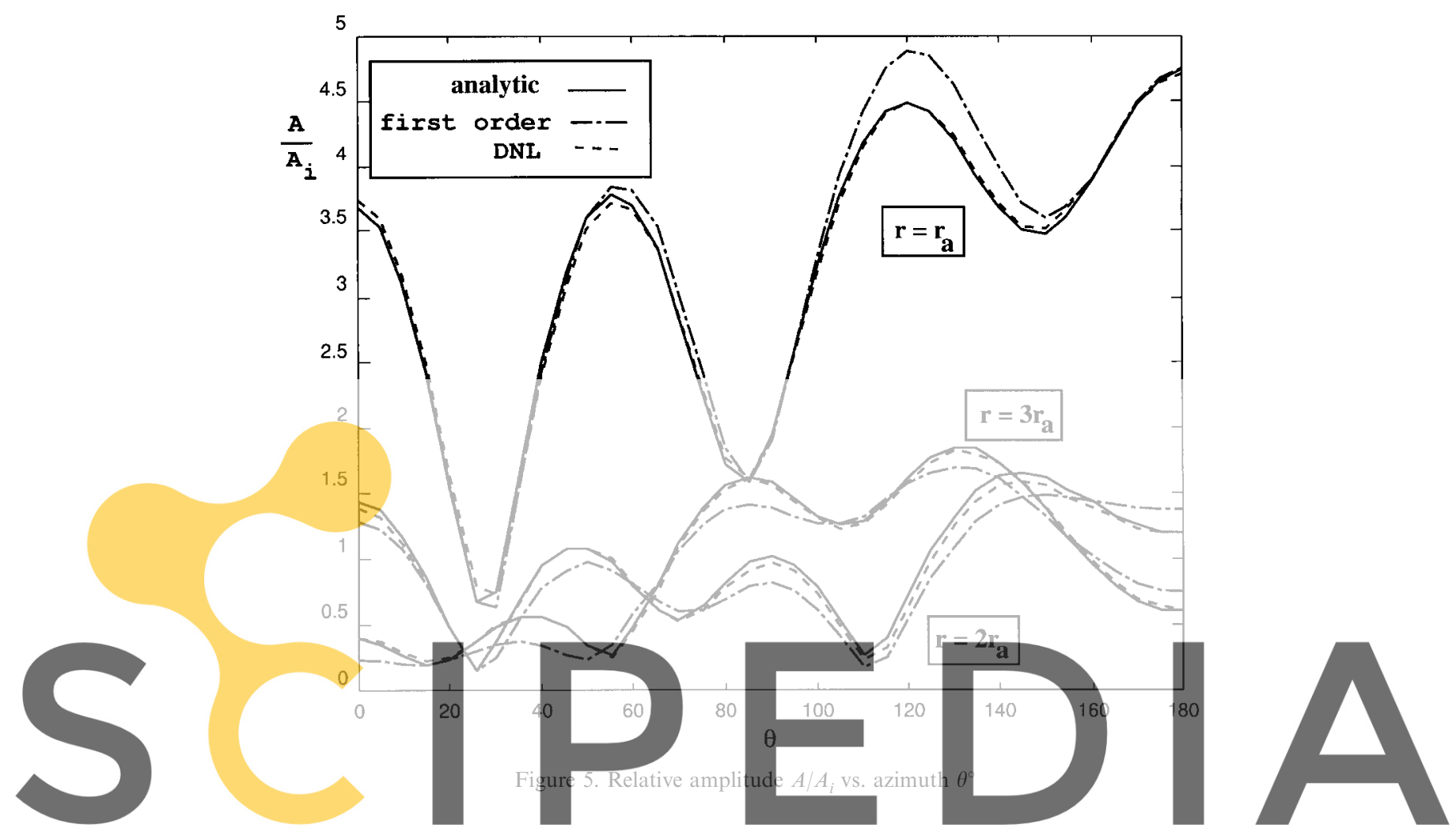

Register for free at http\$//WwW.sqipedia.com to download the'version without the watermark

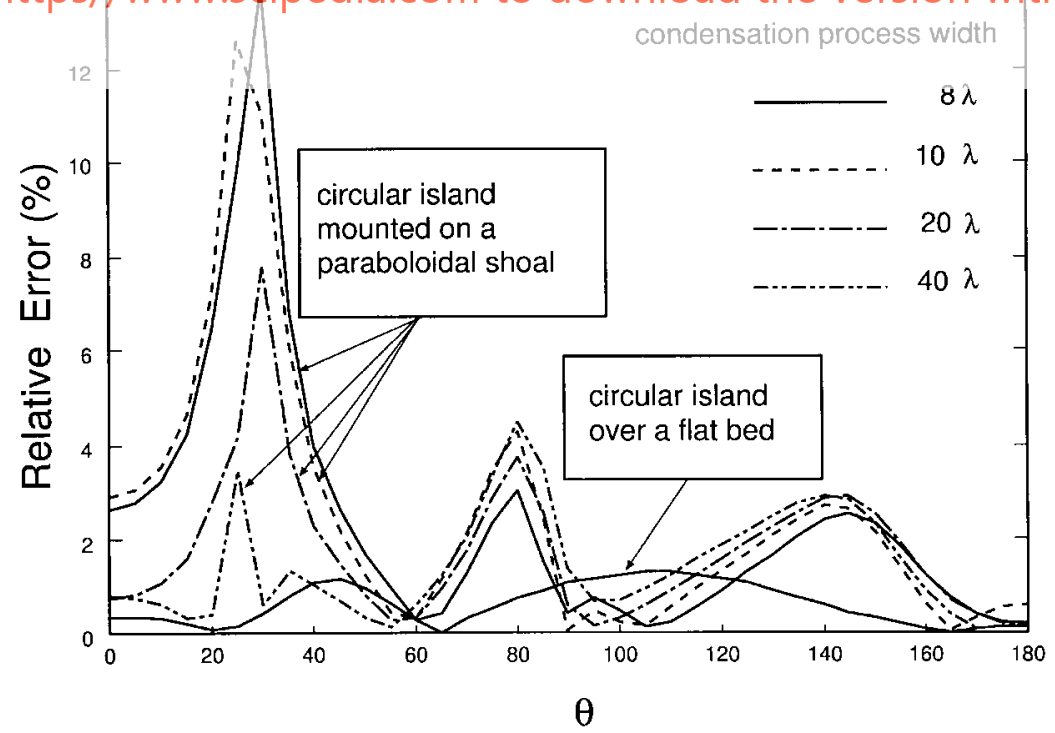

Figure 6. Relative errors at shoreline vs. azimuth $\theta^{\circ}$ 
In the shallow water approximation the diffraction problem has an analytical solution. For this case, Homma ${ }^{1}$ solved the infinite set of linear two-point boundary problems. Figure 5 depicts curves of relative amplitudes $A / A_{i}$ at $r=r_{a}, r=2 r_{a}$ and $r=3 r_{a}$ vs. azimuth $\theta^{\circ}$. The relative amplitudes at the shoreline (with a radius of $r_{a}=10 \mathrm{~km}$ ) correspond to the results reproduced in other papers ${ }^{13,15}$ for a period of $T=240 \mathrm{~s}$. This curve has the same form as the curve obtained by Zienkiewicz et al. for this period, but there is a discrepancy with the relative amplitudes reported by them, due to the difference in the geometrical parameters relative to the parabolic shoal. We note that, for the same period, if $\alpha$ increases, then the relative amplitudes at the island also increase.

The numerical solutions (Figures 4 and 5) by the finite element method and the DNL procedure were obtained when the radius $r=r_{\mathrm{e}}$ is located $8 \lambda$ from the artificial boundary. Figure 6 shows the corresponding relative errors (in per cent) at the island with the continuous right line.

We can observe that the maximum relative errors diminish as the circle $r=r_{\mathrm{e}}$ is placed far away from the computational domain. It is interesting to study how the wave at the island is delayed compared to the far field for the same value of $r$. In other words, the phase delay ('local phase lag') tells us how much the presence of the island plus the shoal has retarded the 'undisturbed' wave field. ${ }^{2}$ This can be expressed at the position $r$ as ${ }^{2}$

$$
\Psi=\phi-k r \cos \theta
$$

where the angle $\phi$ is by definition the phase lag relative to the incident waves in the far field at

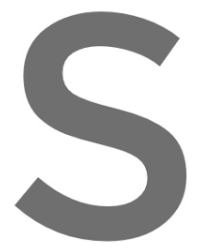

$\theta= \pm 90^{\circ}$. Figure 7
to results obtained by
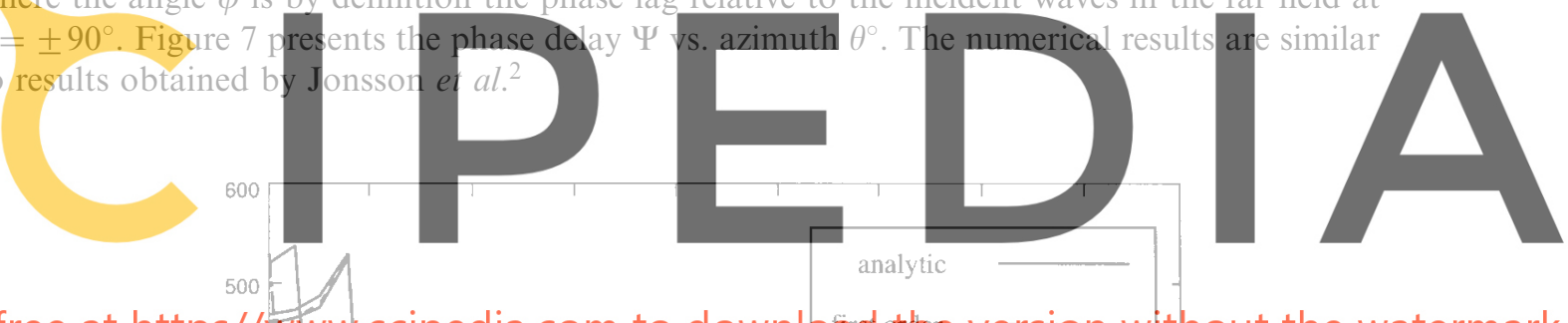

Register for free at https//Www.scipe dia.com to downlobed othe version without the watermark

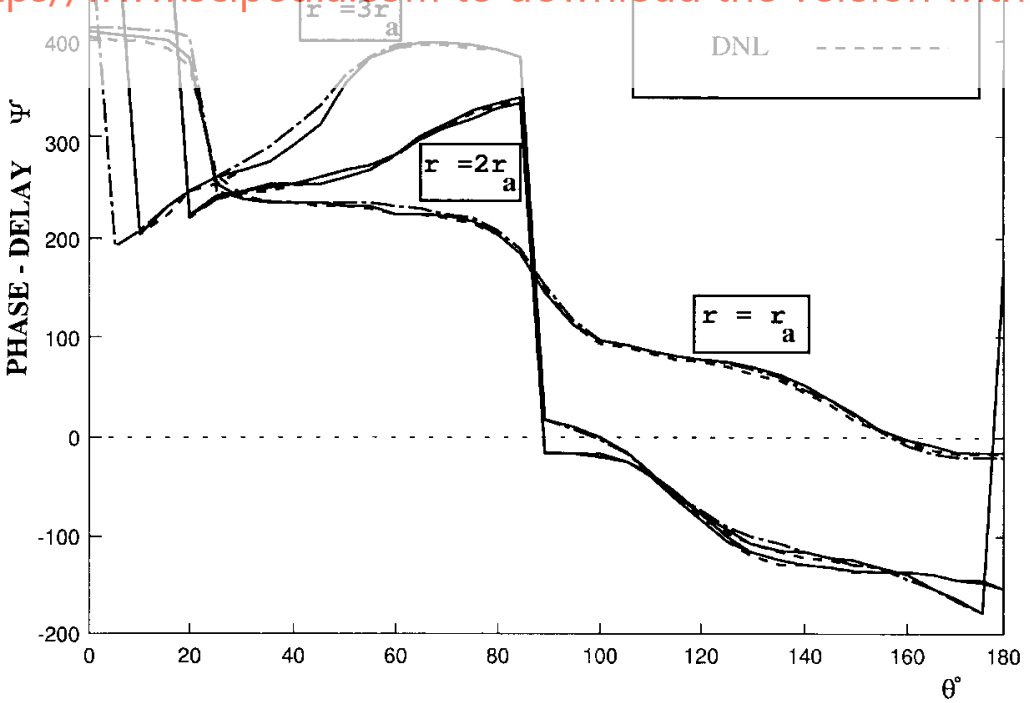

Figure 7. Phase-delay $\Psi^{\circ}$ vs. azimuth $\theta^{\circ}$. Comparison numerical vs. analytical solution based in shallow water diffraction theory 

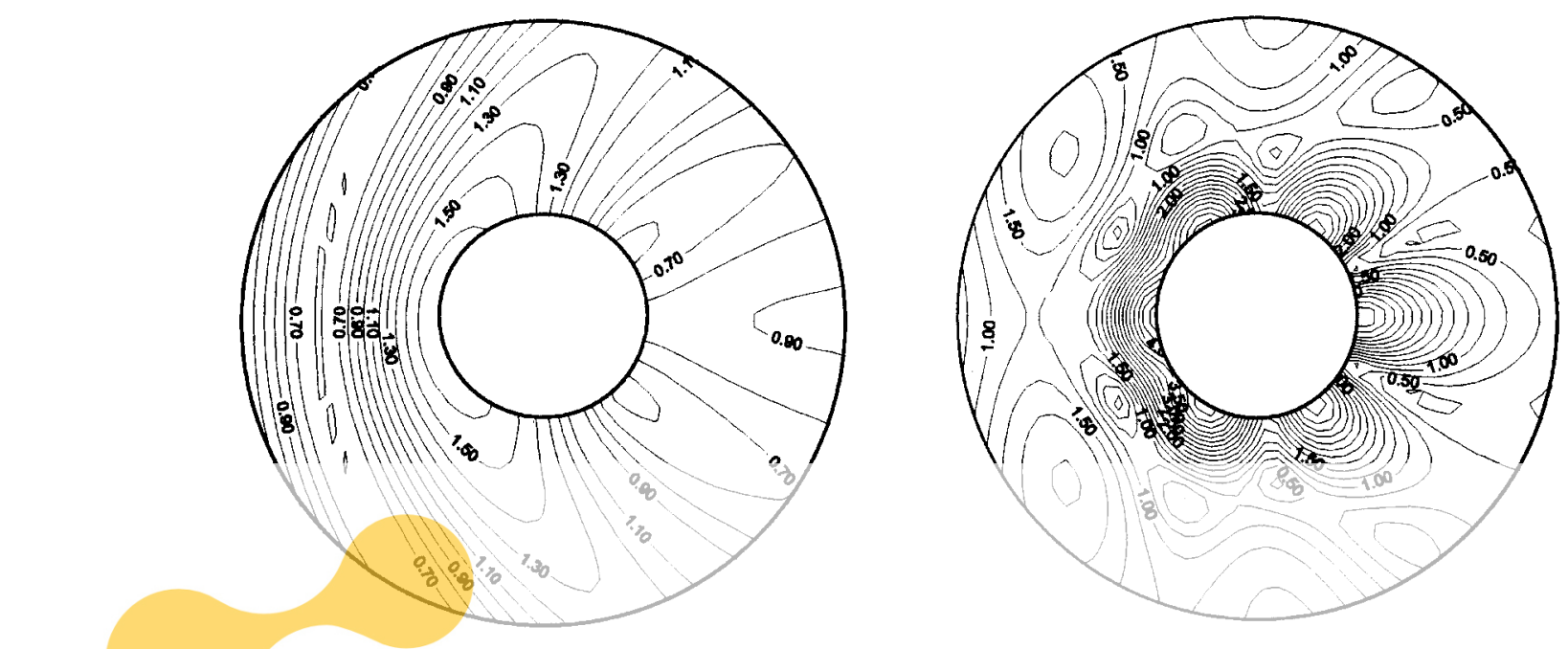

Figure 8. Scattering of a plane wave (at $\theta=0$ ) from a circular island: (left) located on a constant ocean; (right) located on a paraboloidal shoal
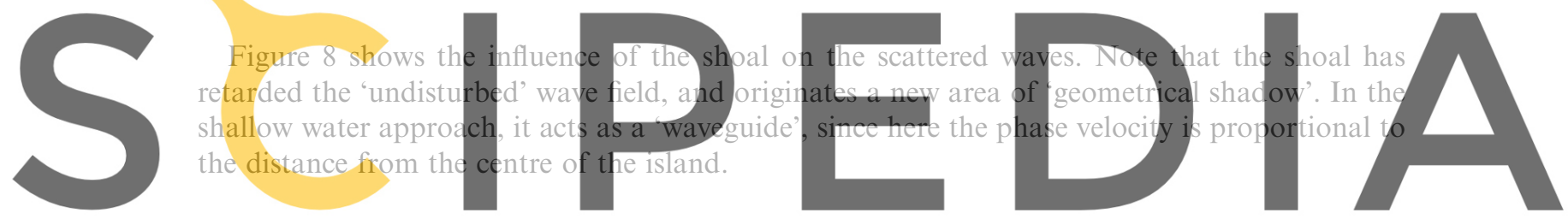

\section{Register for free at https//wym. scipedia com to download}

\section{CONCLUSIONS}

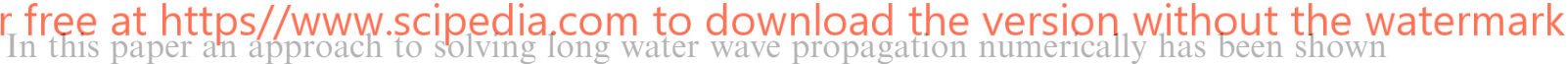

using two diffraction problems as examples. The DNL numerical solution for long waves

scattered by a circular island standing in open sea of constant depth was presented. A novel numerical solution has been developed for water wave propagation in a region of variable water depth. Employing the same condensation width as the previous case, its solution has been obtained. After comparing the results obtained with and without a shoal over the depth, we found that an island located on the paraboloidal shoal induced very strong wave amplifications near the coastline, as expected. The comparison between the numerical result employing the DNL method and the first order local condition shows the improvement of these procedures.

\section{APPENDIX: NOMENCLATURE}

$\mathcal{B}=$ circumference of radius $r_{i}$

$\eta=$ water elevation

$\eta^{\mathrm{s}}=$ scattered field

$\eta_{\mathrm{i}}=$ incoming field (or incoming wave)

$\mathbf{A}_{\mathrm{i}}=$ incoming field (or incoming wave amplitude)

$\Delta=$ Laplacian operator

$k_{0}=2 \pi / L_{0}$ wave number 


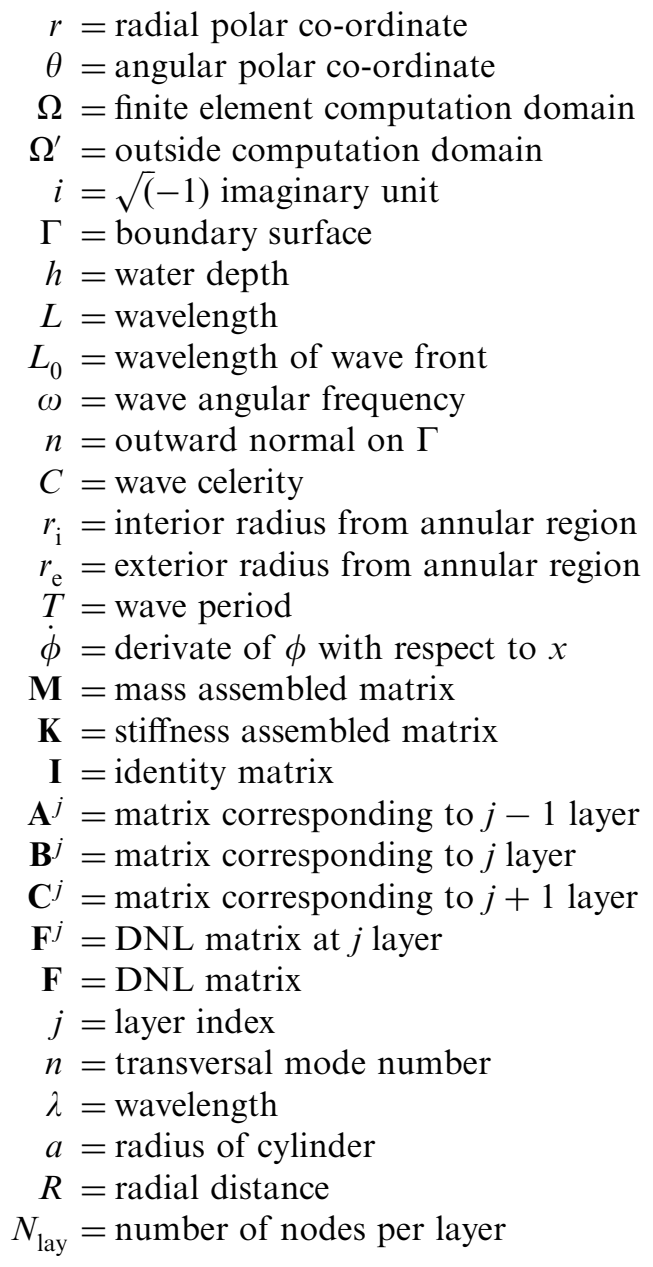

ACKNOWLEDGEMENTS

This work has received financial support from Consejo Nacional de Investigaciones Científicas y Técnicas (CONICET, Argentina) through grant BID 802/OC-AR PID 26, and from Universidad Nacional del Litoral (Argentina). We made extensive use of software distributed by the Free Software Foundation/GNU-Project: Linux ELF-OS, Octave, Tgif from William C. Cheng, Fortran f2c compilers, and others. We also wish to thank the reviewers by their accurate comments and suggestions.

\section{REFERENCES}

1. S. Homma, 'On the behaviour of seismic sea waves around circular island', Geophys. Mag., XXI, 199-208 (1950).

2. I. G. Jonsson, O. Skovgaard and O. Brink-Kjaer, 'Diffraction and refraction calculations for waves incident on an island', J. Mark. Res., 343, $468-496$ (1976). 
3. R. P. Shaw, 'Boundary integral equation methods applied to water waves', $A M D$, Vol. 11, Boundary integral equation method: Computational applications in applied mechanics, 1975, ASME (presented at 1975 Applied Mechanics Conference, the Rensselear Polytechnic Institute, Troy, New York, 23-25 June, 1975, T. A. Cruse and F. Rizzo (Eds.)).

4. A. C. Vastano and R. O. Reid, 'A numerical study of the tsunami response at an island', Dept. of Oceanography, Texas A \& M Univ., A \& Project 471, Ref. 66-26T, 1966, 141 pp.

5. A. C. Vastano and R. O. Reid, 'Tsunami response for islands: Verification of a numerical procedure', J. Mar. Res., 25, 129-139 (1967).

6. O. C. Zienkiewicz, 'The finite element method and boundary solution procedures as general approximation methods for field problems', World Congress on Finite Element Methods in Structural Mechanics, Bournemouth, 12-17 October 1975.

7. O. C. Zienkiewicz, D. W. Kelly and P. Bettess, 'The coupling of the finite element method and boundary solution procedures', Int. j. numer. methods eng., 11(2), 355-375 (1977).

8. O. C. Zienkiewicz, D. W. Kelly and P. Bettess, 'Marriage á la mode - or The Best of Both Worlds. Boundary integrals and finite element procedures', Conf. on Innovative Methods of Numerical Computation, Versailles, France, May 1977.

9. O. C. Zienkiewicz, P. Bettess and D. W. Kelly, 'The finite element method for determining fluid loading on rigid structures: two and three-dimensional formulations', in Numerical Methods in Offshore Engineering, O. C. Zienkiewicz, R. W. Lewis and K. G. Staff (Eds.), Wiley, 1978, Chap. 4.

10. P. Bettess and O. C. Zienkiewicz, 'Diffraction and refraction of surface waves using finite and infinite elements', Int. j. numer. methods eng., 11, 1271-1290 (1977).

11. O. C. Zienkiewicz and P. Bettess, 'Infinite elements in the study of fluid-structure interaction problems', 2nd International Symposium on Computing Methods in Applied Science and Engineering, Versailles, France, 15-17 December 1975.

12. J. C. W. Berkhoff, 'Mathematical models for simple harmonic linear water waves, wave diffraction and refraction', Delft Hydraulic Laboratory, Public. Nro. 163, 1976.

13. H. S. Chen and C. C. Mei, 'Oscillations and wave forces in an offshore harbor: Applications of hybrid finite element method to water-wave scattering', Ralph M. Parsons Laboratory for Water Resources and Hydrodynamics, Mass. Inst. Tech., Rep. No. 190, 1974.

14. H. S. Chen and C. C. Mei, 'Oscillations and wave forces in a man-mode harbor in the open sea', presented at the 10th Naval Hydrodynamics Symposium, June 1974.

15. H. S. Chen and C. C. Mei, 'Hybrid-element method for water waves', Proceedings of the Modelling Techniques Conference (Modelling 1975), Vol. 1, San Francisco, 3-5 September 1975, pp. 63-81.

16. T.-K. Tsay and P. L.-F. Liu, 'A finite element model for wave refraction and diffraction', Appl. Ocean Res., 5, 30-37 (1983).

17. J. R. Houston, 'Combined refraction and diffraction of short waves using the finite element method', Appl. Ocean Res., 3, 163-170 (1981).

18. B. Xu, V. G. Panchang and Z. Demirbilek, 'Exterior reflections in elliptic harbor wave models', J. Waterway Port Coastal Ocean Eng., 122, 118-126 (1996).

19. R. P. Bonet, N. Nigro and M. A. Storti, 'Open boundary conditions for elliptic water wave models (CIMAF97)', La Havana, 24-27 March 1997.

20. H. S. Chen, 'Infinite elements for water wave radiation and scattering', Int. j. numer. methods fluids, 11, 555-569 (1990).

21. D. Givoli and J. B. Keller, 'Non-reflecting boundary conditions for elastic waves', Wave Motion, 12, 261-279 (1990).

22. D. Givoli, Numerical Methods for Problems in Infinite Domains, Elsevier, Amsterdam, 1992.

23. I. Harari and T. J. R. Hughes, 'Studies of domain-based formulations for computing exterior problems of acoustics', Int. j. numer. methods eng., 37, 2935-2950 (1994).

24. R. W. Thatcher, 'On the finite element method for unbounded regions', SIAM J. Numer. Anal., 15(3), 456-477 (1978).

25. R. W. Thatcher, 'The use of infinite grid refinements at singularities in the solution of Laplace's equation', Numer. Math., 25, 163-178 (1976).

26. G. Dasgupta, 'Sommerfeld's radiation condition and cloning algorithm', ASCE-ASME Summer Conference, Boulder, CO, 1981. 
27. Ch. Song and J. P. Wolf, 'Consistent infinitesimal finite-element cell method: Three dimensional vector wave equation', Int. j. numer. methods eng., 39, 2189-2208 (1996).

28. J. P. Wolf and Ch. Song, Finite Element Modelling of Unbounded Media, Wiley, 1996, $331 \mathrm{pp}$.

29. R. P. Bonet, N. Nigro, M. A. Storti and S. R. Idelsohn, 'Non-local absorbing discrete boundary condition (DNL) in finite difference for water waves elliptic models' (in Spanish; submitted to RIMNE, 1997).

30. R. P. Bonet, N. Nigro, M. A. Storti and S. R. Idelsohn, 'Non-local absorbing discrete boundary condition (DNL) in finite elements for water waves elliptic models' (in Spanish; submitted to RIMNE, 1997).

31. R. P. Bonet, N. Nigro, M. A. Storti and S. R. Idelsohn, 'Discrete non-local absorbing discrete boundary condition for exterior problems governed by Helmholtz equation' (submitted for publication to Int. j. numer. methods fluids, 1997).

32. M. A. Storti, J. D.'Elía and S. R. Idelsohn, 'Algebraic discrete non-local (DNL) for the ship wave resistance problem' (submitted for publication to J. Comput. Phys., 1997).

33. R. P. Shaw, 'Transient scattering by a circular island', J. Sound Vib., 42(3), 295-304, (1975). 